and individual levels are required as well as reducing prevalence of risk factors across the whole population.

\section{RF35 SUPPORT THAT COMES FROM CULTURE: A RASCH ANALYSIS OF THE SOCIAL SUPPORT SCALE (SSS) IN AN ABORIGINAL POPULATION}

${ }^{1}$ PHR Santiago*, ${ }^{2} \mathrm{~L}$ Smithers, ${ }^{3} \mathrm{R}$ Roberts, 'L Jamieson. 'Adelaide Dental School, The University of Adelaide, Adelaide, Australia; ${ }^{2}$ School of Public Health, The University of Adelaide, Adelaide, Australia; ${ }^{3}$ School of Psychology, The University of Adelaide, Adelaide, Australia

\subsection{6/jech-2019-SSMabstracts. 150}

Background Due to a history of colonization, Aboriginal and Torres Strait Islander became one of the most disadvantaged groups in Australia. The decades of assimilation policies, which culminated in the removal of Aboriginal children from their parents, disarranged the social support derived from their kinship system. In non-Indigenous populations, social support has been reported as a protective factor against stress (i.e. buffering hypothesis) and coronary heart disease; providing also better outcomes for breast cancer and type- 2 diabetes. There are no psychological instruments validated to measure social support in Aboriginal Australians. The aim of the current study was to evaluate the validity and reliability of the Social Support Scale in an Aboriginal population.

Methods The Social Support Scale (SSS) is a 4-item measure developed to evaluate the emotional, appraisal, instrumental and informational domains of social support. Data was collected from to two different samples: participants of the (1) Teeth Talk Study $(n=317)$, an oral-health randomized controlled trial (RCT) conducted among Aboriginal adults; and (2) the Baby Teeth Talk Study $(n=367)$, an RCT conducted among pregnant Aboriginal women. The psychometric properties of the SSS were evaluated with Graphical Loglinear Rasch Models (GLLRM), which consist of recent methodological advances that extend the Rasch Model to incorporate local dependence (LD) and differential item functioning (DIF).

Results Overall fit to a GLLRM was found $\left(X^{2}(96)_{\text {sample } 1}=52.7\right.$, $\left.\mathrm{p}=0.057 ; \mathrm{X}^{2}(25)_{\text {sample } 2}=22.2, \mathrm{p}=0.625\right)$ after the inclusion of LD between items 3 and $4\left(g_{a v g 1}=0.50 ; g_{a v g 2}=0.66\right)$. Item 2 displayed DIF by employment status $\left(\mathrm{X}^{2}(4)=12.60, \mathrm{p}=0.013\right.$, $g=0.21)$ in Sample 1. There was no DIF by sex or educational attainment. Unidimensionality was confirmed in both samples $\left(\mathrm{g}_{\mathrm{obs} 1}=0.80 ; \quad \mathrm{g}_{\exp 1}=0.78, \mathrm{p}=0.654 ; \mathrm{g}_{\mathrm{obs} 2}=0.75, \mathrm{~g}_{\exp 2}=0.77\right.$, $\mathrm{p}=0.163)$. The SSS displayed good reliability $\left(\mathrm{R}_{\text {sample } 1}=0.82\right.$, $\left.\mathrm{R}_{\text {sample } 2}=0.84\right)$ and probability of person separation $\left(\mathrm{P}_{\text {sample } 1}=0.77\right.$, $\left.\mathrm{P}_{\text {sample } 2}=0.78\right)$. Targeting was poor $\quad\left(\mathrm{TTI}_{\text {sample } 1}=0.28\right.$; $\mathrm{TTI}_{\text {sample2 }}=0.49$ ).

Conclusion The same unidimensional structure was found in both samples, providing confidence in the robustness of results. Item 2 ('There are people in my life who appreciate what I do') displayed DIF by employment status, indicating that participants who were employed were more likely to feel appreciated than those unemployed given the same level of social support. The current study consisted of the first validation of a Western-developed psychological instrument to measure social support in Aboriginal Australians. The results show that the SSS is a culturally-valid and reliable instrument that can be applied in future health research among Aboriginal and Torres Strait Islanders.

\section{RF36 COMPARING MULTIPLE FORMS OF DISCRIMINATION AND POSTPARTUM DEPRESSION AMONG PALESTINIAN-ARAB MINORITY WOMEN, IMMIGRANT AND NON-IMMIGRANT JEWISH WOMEN IN ISRAEL}

${ }^{1} \mathrm{~N}$ Daoud*, ${ }^{1} \mathrm{R}$ Sergienko, ${ }^{2} \mathrm{M}$ Geo. 'Department of Public Health, Ben-Gurion University of the Negev, Beer-Sheva, Israel; ${ }^{2}$ Critical Care Services, Critical Care Services, Toronto, Canada

\subsection{6/jech-2019-SSMabstracts. 151}

Background Drawing on the intersectionality theory, emerging research shows that discrimination is a multidimensional risk factor for health. Yet, associations between multiple forms of discrimination (MFD) and postpartum depression (PPD) have not been explored. This study compares relationships between MFD and PPD among Palestinian-Arab indigenous minority, Jewish immigrant, and Jewish non-immigrant mothers citizens of Israel.

Methods We used data from a stratified sample of 1,128 postpartum mothers who were interviewed during visiting maternal and child health clinics in 2014-15. We conducted multivariable logistic regression analysis and generalized estimation equation for PPD (Edinburgh Postnatal Depression Scale with cutoff $\geq 10$ ) and compared associations with two measure of MFD among the study groups, while considering age, socioeconomic status, anti-depressant use, and single forms of discrimination in different models. The two MFD measures included: cumulative MFD (additive experiences of $0,1,2$ or $\geq 3$ forms of discrimination based on ethnicity, skin color, religiosity level, gender, age and socioeconomic status), and composite MFD (12 categories resulting from an interactions terms between cumulative $0,1,2$ and $3 \geq$ MFD and women's study groups. The reference category was non-immigrant Jewish mothers who reported no MFD.

Results Palestinian-Arab mothers reported highest MFD, followed by Jewish immigrant mothers and non-immigrant Jews $(\geq 3 \mathrm{MFD}=29.2 \%, 24.1 \%$ and $17.8 \%$, respectively). Composite MFD had stronger dose response associations with PPD among Palestinian-Arab women, followed by immigrant Jews and non-immigrant Jewish women. Compared to non-immigrant Jewish women with no MFD, Palestinian-Arab women reporting $\geq 3 \mathrm{MFD}, 2 \mathrm{MFD}$ and one MFD were more likely to experience PPD. Adjusted odds ratio and 95\% confidence intervals (AOR, 95\%CI) were 12.68 (5.29-30.40), 10.08 (3.73-27.20), and 3.98 (1.23-12.86), respectively, among Palestinian-Arab women; 4.44 (1.45-13.61), 5.76 (1.84-17.97), 2.32 (0.59-9.12), respectively, among immigrant Jewish mothers; and 4.68 (1.87-11.71), $3.74(1.32-10.63)$ and 2.70 (1.06-6.87) among non-immigrant Jews mothers. Cumulative or additive MFD showed a strong dose response association with PPD among non-immigrant Jews and Palestinian-Arab women who reported $\geq 3 \mathrm{MFD}$, but not among immigrant Jewish women.

Conclusion The study result sheds light on the importance of studying the facets of MFD in intersection with social 
identities in maternal mental health research. Using a cumulative or additive measure of MFD might underestimate the association between discrimination and PPD specifically in minority and immigrant mothers who face MFD. Health care providers should consider the effects of MFD on PPD among mothers, especially for women located at more than one marginalized axis of identity.

\section{RF37 PREDICTORS OF ATTITUDES TOWARDS PEOPLE WITH DISABILITY IN AUSTRALIA: FINDINGS FROM A CROSS-SECTIONAL SURVEY OF AUSTRALIAN ADULTS}

A Kavanagh*, AM Bollier, L Krnjacki, G Katsikis, V Kasidis, J Ozge, A Milner. Melbourne School of Population and Global Health, University of Melbourne, Melbourne, Australia

10.1136/jech-2019-SSMabstracts. 152

Background There have been many studies on attitudes to disability, but few are nationally representative, and little is known about how attitudes vary by socio-demographic characteristics and contact with disabled people.

Methods We used data from 2,000 members of an Australian probability panel who completed a survey on disabilityrelated attitudes. Attitudes were measured with the Attitudes to Disability Scale (ADS), which asks 16 questions in 5point Likert-type format. The outcome for this analysis was the Prospects sub-scale, which asks whether the capacities and aspirations of disabled people should differ from those of non-disabled people. Raw scores out of 20 points were standardized (mean 0, SD 1) with positive scores representing more positive attitudes. Sociodemographic variables (disability/carer status, age cohort, education level, country of birth, socio-economic disadvantage, gender, and region of residence) were fitted in a linear regression model to examine predictors of attitudes. Eight measures of lifetime contact with people with disability (e.g. ever lived with a person with disability [yes/no]) were then fitted in separate models to examine associations with attitudes, adjusting for potential confounders excludes participants with a disability and carers.

Results Female (versus male) gender predicted more positive attitudes $(\beta=0.36,95 \%$ CI $0.23,0.49)$, as did overseas English-speaking origin $(\beta=0.20,95 \%$ CI $0.02,0.39)$ relative to native Australian, and year 12 educational attainment $(\beta=0.41,95 \%$ CI $0.21,0.60)$ or university study $(\beta=0.39$, $95 \%$ CI $0.20,0.58)$ relative to less than year 12 . People with disability (versus non-disabled non-carers) had more negative attitudes $(\beta=-0.15,95 \%$ CI $-0.30,-0.01)$, as did members of the oldest (Lucky) generation versus the youngest (iGeneration) ( $\beta=-0.37,95 \%$ CI $-0.62,-0.12)$ and overseas non-English speaking origin $(\beta=-0.37,95 \%$ CI -0.62 , -0.13) versus native Australian. In the 8 contact models, 4 were associated with more positive attitudes: ever having a colleague $(\beta=0.29,95 \%$ CI $0.13,0.45)$; classmate $(\beta=0.26$, $95 \%$ CI $0.09,0.43)$; close friend $(\beta=0.25,95 \%$ CI 0.07 , $0.42)$; and teacher/boss $(\beta=0.27,95 \%$ CI $0.02,0.52)$ with disability.

Conclusion This study is the first Australian population-based study of predictors of attitudes to disability. Internalized stigma might explain why disability was associated with more negative attitudes among disabled people. Because this is a cross-sectional study, we do not know the direction of the relationship between having friends with disability and positive attitudes however our findings suggest that peer relationships in social environments and contact in work and educational settings promote positive attitudes to disabled people.

\section{Poster Programme}

\section{P01 CHILDHOOD SOCIO-ECONOMIC POSITION AND MIDLIFE PHYSICAL FUNCTIONING: EXPLORING ASSOCIATIONS AND UNDERLYING MECHANISMS IN THE 1958 BRITISH BIRTH COHORT}

A Ahmed* , SP Pereira. Epidemiology and Public Health, University College London, London, UK

10.1136/jech-2019-SSMabstracts. 153

Background Evidence suggests that childhood socioeconomic position (CSEP) is associated with adult physical functioning $(\mathrm{PF})$. However, literature is limited on key life-course intermediaries that explain this relationship. In the 1958 British birth cohort, we aimed to examine (1) the association between CSEP and PF in midlife, and (2) potential adult intermediaries (i.e. social, psychological, behavioural and biological pathways) that could explain the CSEP-PF relationship.

Methods CSEP recorded at birth (or at 7y) was represented by 4 categories of father's occupational class (professional/ managerial, skilled non-manual, skilled manual and partly skilled/unskilled). PF was assessed at $50 \mathrm{y}$ using the validated PF subscale of the SF-36 questionnaire. Participants with a score at or below the gender-specific 10th-centile were classified as having poor PF. Prospectively collected data represented each of the four potential intermediary pathways: social (42y socioeconomic position, 33y education), psychological (42y mental health), behavioural (42y smoking, drinking and physical activity) and biological (45y measured BMI, waist-hip ratio, blood pressure, lung function, HbA1c and blood lipids). Gender specific associations were examined using logistic regression, in STATA v15. Adjustments were made for prospectively recorded early-life covariates (birthweight, maternal smoking, childhood disability and presence of childhood illnesses).

Results In unadjusted analysis ( $\mathrm{n}=4056$ men, $\mathrm{n}=4375$ women) odds ratios (ORs) for poor PF increased with decreasing CSEP. This trend was maintained after covariate adjustment e. g. in women, compared to the professional/managerial class, adjusted-OR for skilled non-manual was 1.31[0.77,2.24]; skilled manual was $1.86[1.28,2.69]$ and partly skilled/unskilled was 2.23[1.49,3.35]. After accounting for the social pathway, associations were markedly attenuated, particularly in women; e.g. compared to the professional/managerial class OR for skilled non-manual was $0.87[0.46,1.68]$; skilled manual was $1.24[0.80,1.93]$ and partly skilled/unskilled was 1.30 $[0.79,2.14]$. Behavioural factors attenuated associations to a lesser extent. Biological factors completely attenuated associations in women, whereas associations remained in men. Associations were little affected by psychological factors.

Conclusion While there was a trend between lower CSEP and higher risk of poor midlife PF in men and women, intermediary pathways differed by gender. Better adult education and employment opportunities may help alleviate the burden of low CSEP for all, however improving biomedical profiles may 